VLSI DESIGN

1996, Vol. 4, No. 1, pp. 69-74

Reprints available directly from the publisher

Photocopying permitted by license only
(C) 1996 OPA (Overseas Publishers Association) Amsterdam B.V.

Published in The Netherlands under license by Gordon and Breach Science Publishers SA Printed in Malaysia

\title{
Integration of SPICE with TEK LV500 ASIC Design Verification System
}

\author{
A. SRIVASTAVA and S. R. PALAVALI \\ Department of Electrical and Computer Engineering, Louisiana State University, Baton Rouge, LA 70803
}

(Received April 13, 1993, Revised June 22, 1994)

\begin{abstract}
The present work involves integration of the simulation stage of design of a VLSI circuit and its testing stage. The SPICE simulator, TEK LV500 ASIC Design Verification System, and TekWaves, a test program generator for LV500, were integrated. A software interface in 'C' language in UNIX 'solaris1. $x$ ' environment has been developed between SPICE and the testing tools (TekWAVES and LV500). The function of the software interface developed is multifold. It takes input from either SPICE2G.6 or SPICE 3e.1. The output generated by the interface software can be given as an input to either TekWAVES or LV500. A graphical user interface has also been developed with OPENWINDOWS using Xview tool kit on SUN workstation. As an example, a two phase clock generator circuit has been considered and usefulness of the software demonstrated. The interface software could be easily linked with VLSI design such as MAGIC layout editor.
\end{abstract}

Key Words: Testability, SPICE, ASIC, CAD Tools, Design

\section{INTRODUCTION}

The development of a VLSI circuit involves many steps such as circuit design, layout design, simulation, fabrication and testing $[1,2]$. For testing a digital system implemented in a VLSI circuit, we need a set of input stimulus vectors which are forced on the input pins of the VLSI circuit, and the expected output response from the system which is used to compare with the actual response of the VLSI circuit being tested [3,4].

The input stimulus vectors and the netlist extracted from the VLSI circuit layout are given to a simulator. The simulator simulates the circuit in hand using the circuit definition file which consists of device equivalent circuit models and other network parameters [5,6]. The simulator, then runs analysis for the circuit definition file and gives the output response corresponding to a defined set of DC and/or pulsed binary input parameters. These DC and/or pulsed binary input parameters and corresponding generated output response by the simulator are termed as input and output vectors. The vectors needed for testing are thus generated by simulation but these vectors are in a format specific to the simulator used. The tester may need them in a different format with additional information such as clocking information [7]. All these needs are to be provided by the testing engineer. This process consumes time and is error prone since human factor is involved [8].

The design automation and testing process of a VLSI circuit through an interface between a simulator and a design verification system enhances the efficiency of testing since the involvement of human factor is greatly reduced. The automation of testing process also reduces the time consumed since the test program for the VLSI tester is generated by the interface.

\section{CAD TOOLS SELECTED FOR INTEGRATION}

The design verification system consists of "TEK LV500 ASIC Design Verification System" and "TekWAVES", respectively. The LV500 is a stand-alone design verification tester for application specific integrated circuits [9]. It provides 64 to 256 bidirectional tester channels at test speeds up to $50 \mathrm{MHz}$. In this work, the tester used provides 64 channels. The LV500 takes input stimulus vectors and expected output vectors for a particular integrated circuit under test in the form of a test program. It tests the chip mounted on it by comparing the actual output vectors obtained from a fabricated IC chip and the expected output vectors fed to it, and shows the discrepancies between the two, if any. The TekWAVES is a 
software package by which digital stimulus vectors can be created, viewed and manipulated [10]. It aids LV500 in ASIC design verification. With TekWAVES, the test programs can be acquired and downloaded to and from LV500. The TekWAVES needs the input vectors and expected output vectors in an event wave (EWAV) format as an input. The EWAV format is explained in Section 3.

The simulation tool SPICE [6] is most commonly used for circuit simulation. It takes the input vectors and the netlist extracted from an IC layout in a code specific to it as the inputs. The SPICE simulation is run on the SPICE input file and the output vectors are generated and printed out into an output file as shown by an example in Appendix A. It is to be mentioned that the format of the output file of SPICE3e.1 differs from that of SPICE2G.6. The hierarchical structure and data flow diagram of the present work are shown in Figs. 1 and 2, respectively.

\section{SOFTWARE INTERFACE DEVELOPMENT AND IMPLEMENTATION}

The software interface has been developed using ' $C$ ' language in UNIX 'solaris1.x' environment. The input pulses needed are taken from input files of SPICE simulator and simulated output pulses are extracted from SPICE output.

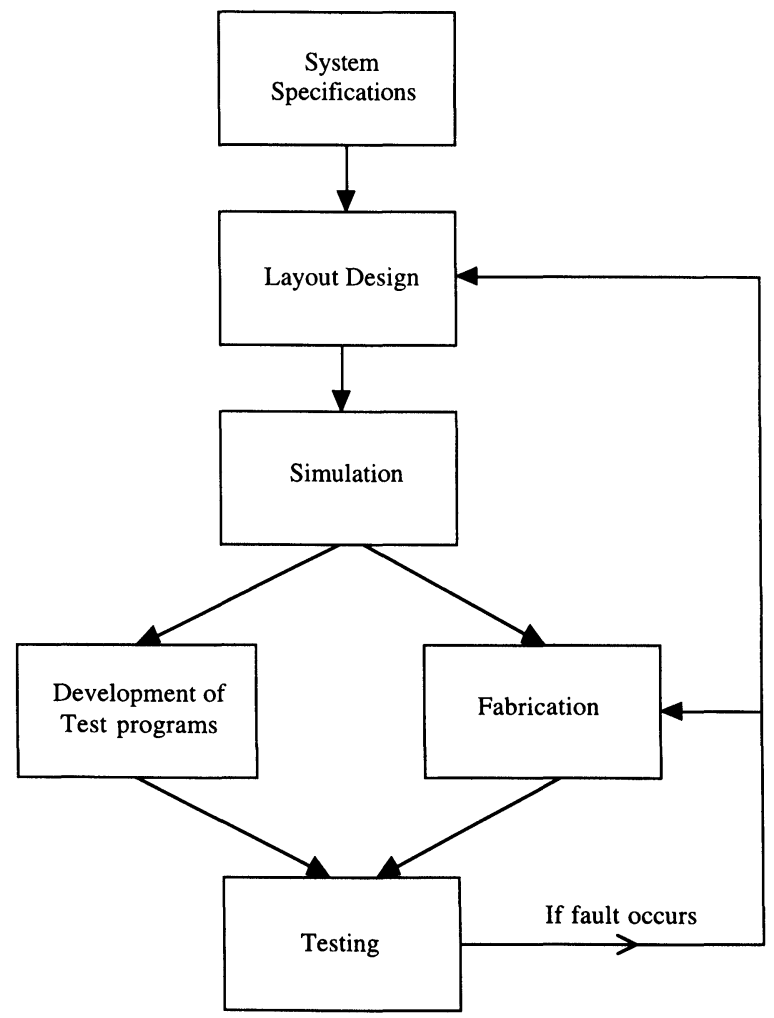

FIGURE 1 Hierarchy of processes in VLSI chip development.

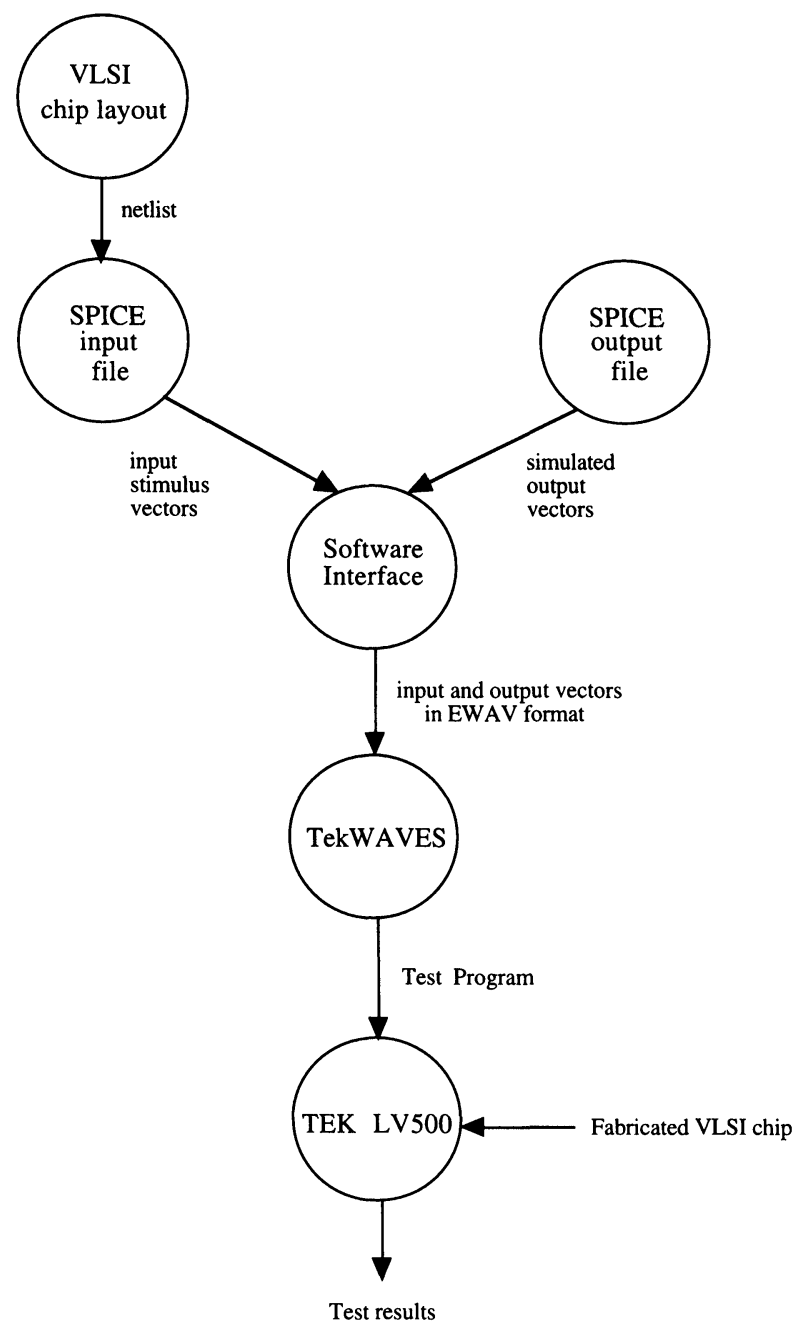

FIGURE 2 Data flow diagram.

All the formats of input vectors that result in generation of digital pulses are converted into EWAV (event wave) format. The output pulses corresponding to various output nodes specified in SPICE input file are found in SPICE output file, and converted into EWAV format.

\subsection{EWAV File Format}

The EWAV file format is an ascii format for event files for logic simulators and testers. The EWAV file format has three sections [10]. The first section is an 'environment' section which contains information such as EWAV file version, the data created, the time created, the intended destination, and the time seale. The second section is the 'signal declaration' section which contains a list of signal definitions. The final section is the 'event data' section which consists of time stamps and the corresponding data vectors. The format of an EWAV file is shown in Fig. 3. 
The Environmental Section:

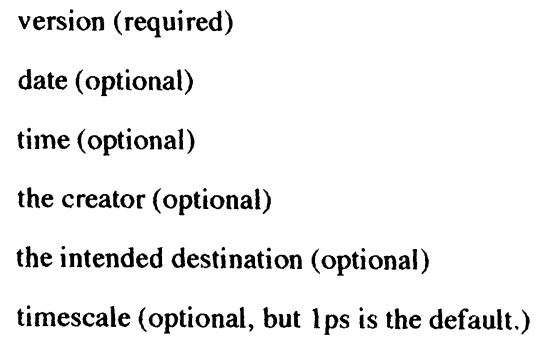

The Signal Declaration Section:

signal name_of_the_signal directionality \{ path (optional)

polarity (optional)

\}

The Event Data Section:

timestamp: event data; (for timestamped data vectors) timestamp@event data with signal keys specified for changed signals; (for timestamped signal change list)

FIGURE 3 EWAV file format.

\subsection{Key Stages in Software Development}

The key stages in software development and the approach followed in each stage with algorithms and pseudo-code are explained in the following subsections through a data flow diagram of Fig. 2.

1. Read the input files (SPICE input and SPICE output files) and locate the statements in the SPICE code that give rise to generation of input vectors.

The pulse and piecewise linear (PWL) statements as shown in Fig. 4 are followed by the specifications for pulses to be generated. These are the statements to be considered in reading input files.

2. Locate PULSE statements and extract the relevant information which includes the pulse width (pw), period (per), and starting voltage (v1) for the vector and the generation of pulses, as an example, PULSE $\left(v_{1}, v_{2}, t_{d}, t_{f}, p_{w}\right.$, per).

It is to be noted that the rise time $\left(t_{r}\right)$ and fall time $\left(t_{f}\right)$ values in the PULSE statement are ignored. This is due to the fact that in the testing of an integrated circuit using TekWAVES and LV500, digital stimulus vectors are given as an input and
*Title line

vdd 10 DC 5

vin1 20 pulse $(\mathrm{v} 1, \mathrm{v} 2, \mathrm{td}, \mathrm{tr}, \mathrm{tf}, \mathrm{pw}, \mathrm{per})$

other statements.

$\operatorname{vin} 230 \mathrm{pwl}(\mathrm{t} 1, \mathrm{v} 1, \mathrm{t} 2, \mathrm{v} 2, \mathrm{t} 3, \mathrm{v} 3, \ldots \ldots \ldots)$

other piecewise linear (pwl) statements.

The extracted netlist goes here

.tran time_step end_time start_time

.print output nodes (for SPICE2G.6)

.save output nodes (for SPICE3e.1)

.end

FIGURE 4 SPICE input file format.

hence the rise time and fall time for these pulses are taken as zero.

3. Locate PWL statements and extract the relevant information, i.e., the time instances and the voltage values at these instances required for the generation of pulses, as an example, PWL $\left(t_{1}, v_{1}, t_{2}\right.$, $\left.\mathrm{v}_{2}, \ldots \ldots \ldots . . . . ..\right)$.

4. Generate input pulses using the parameters in PULSE statements.

5. Generate pulses using the parameters in the PWL statements.

6. Locate the output nodes in SPICE input file.

.PRINT statement in SPICE code has been utilized for this purpose, the reasons being that the statement prints out the voltage values for the nodes specified.

7. Generate the simulated output vectors using the data in SPICE output file.

8. Print the input and output pulses in EWAV format.

The output file is shown as EWAV file in Appendix A. The parameter, for example the time scale, is printed first into an output file (with an extension .ewv). The default time scale for TekWAVES in EWAV format is picoseconds. The time scale is detected from the time instances specified in the PULSE and piece wise linear (PWL) statements, and is printed into the EWAV file if the time scale is not in picoseconds.

The input and output nodes are printed along with the specification of the direction of the node (input, output, 
or bidirectional). The time instances and pulses corresponding to these time instances are printed according to EWAV format.

The vectors in EWAV format are given as an input to TekWAVES. All the necessary steps including generating cycle boundaries, extracting time sets, pin number allocation, LV500 resource allocation, rules check, and finally the wire guide processing are followed with the help of TekWAVES software, in order to extract the test program necessary for LV500.

The data file containing test vectors in EWAV format is tranferred to LV500 through a network communication (ethernet). The chip to be tested is mounted on LV500. The input vectors are passed to the integrated circuit under test. The output digital pulses obtained from the integrated circuit are compared with the simulated output pulses that are present in the test program. The differences between these two are found and reported.

The input vectors to test logic devices can be obtained from their corresponding truth tables. The input vectors can also be generated from the behavioral testing of digital circuits $[11,12]$ at a high level of abstraction which includes fault modeling. This test generation method is basically split into four phases: manifestation phase, sensitization phase, propagation phase, and justification phase. The sensitization and justification sequences constitute the test pattern. However, the present work uses input vectors which could be obtained either form the truth table of a logic device under test or from other test pattern generation techniques. As an example, a two phase clock generator circuit shown in Fig. 5 has been considered. The circuit description is given to SPICE simulator along with the input vectors. For the example shown in Fig. 5, the test vectors were generated form the truth table of a two phase clock generator and were specified both in the PULSE and PWL formats of SPICE simulator. The SPICE2G.6 simulation is run on the circuit and the results of the simulation are shown in Appendix A (SPICE2G.6 output file). The first section of EWAV file generated in Appendix A is the environmental section in which the data, time, and intended destination are optional, but the version at the beginning and the time scale at the end of the section are required. The time scale, if not specified, defaults to ' 1 picosecond.' The second section shows the list of input and output signals along with the respective polarities. The third section

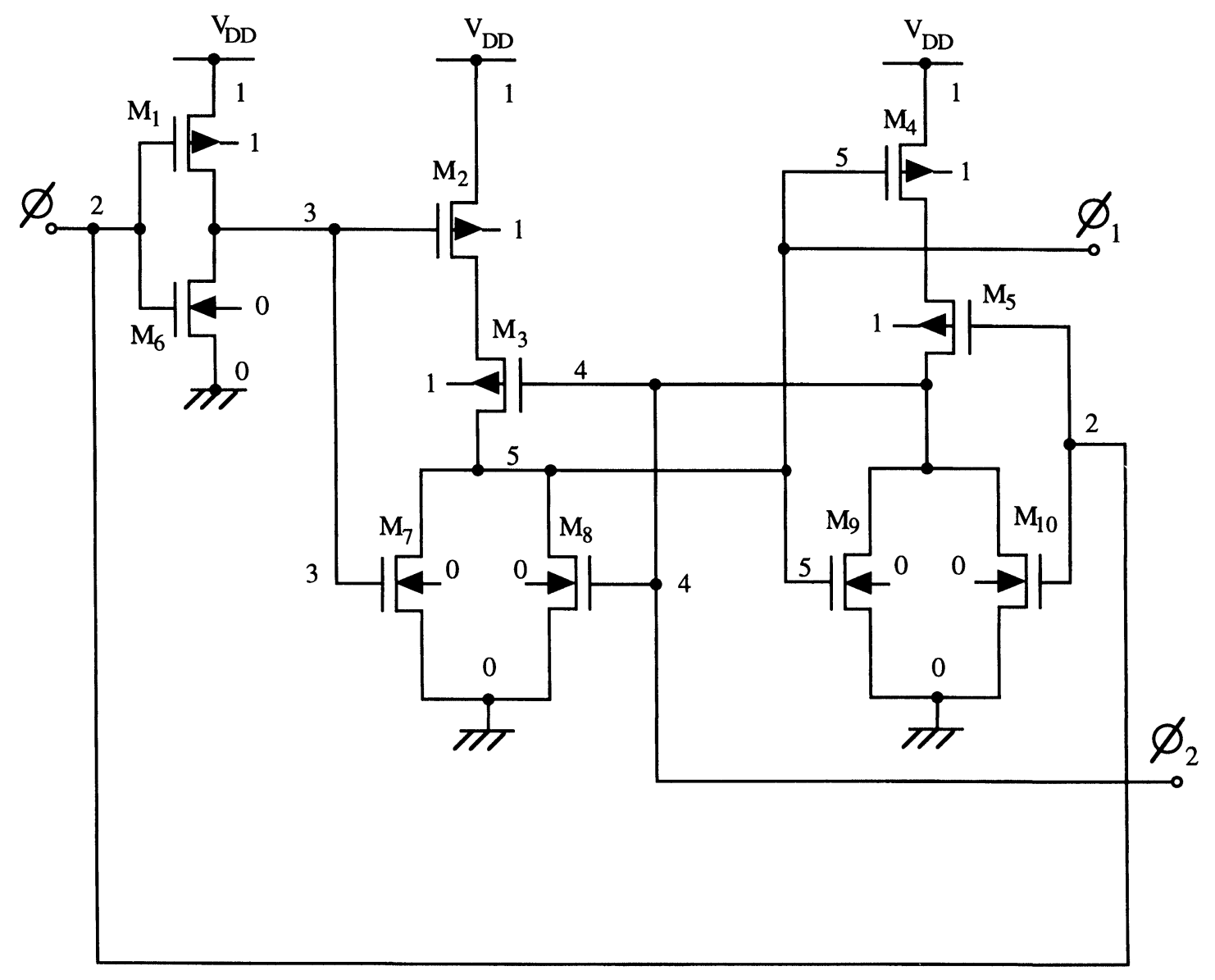

FIGURE 5 Transistor level diagram of a CMOS two phase non-overlapping clock generator. 
provides the time stamps to the left and the data vectors in columns to the right.

The SPICE input file and each of the SPICE output files (one at a time) are given now as inputs to the software interface and the results are written into EWAV file, which are shown as EWAV file in Appendix A.

\subsection{Advantages of the Interface}

The interface developed between a simulator and design verification system enhances the efficiency of testing by reducing the involvement of human factor. The test engineer, now does not need to feed the test vectors by hand to the tester. Further, the test program with clocking and frame information is generated by the interface. Thus, the interface reduces the time consumed in design verification of digital systems.

By virtue of the interface, details such as changing the format of test vectors and including the clocking information in the test program are abstracted away from the user. The user can see the process starting from simulation to testing as a black box, providing the test vectors through simulation in the beginning and running tests on the chip at the end.

\section{CONCLUSIONS}

An interface software in ' $\mathrm{C}$ ' language has been developed to integrate SPICE netlist from VLSI circuit layout (along with the test vectors) and TEK LV500 ASIC Design Verification System through TekWAVES. The output generated by the software can be given as an input to either TekWAVES or TEK LV500. The formats of both SPICE2G.6 and SPICE3e.1 have been incorporated in $\mathrm{CAD}$ tools integration. A graphical user interface has been developed for its efficient use. The utility of the software has been demonstrated through the design of a two phase non-overlapping clock generator circuit.

\section{Acknowledgements}

Authors are very grateful to the reviewers for their valuable comments and suggestions. The partial support of the present work was provided by an equipment research grant from the Tektronix Foundation and is thankfully acknowledged.

\section{References}

[1] C. Mead and L. Conway, Introduction to VLSI Systems, Addison-Wesley, CA, 1980.

[2] N.H. Weste and K. Eshraghian, Principals of CMOS VLSI Design, Addison-Wesley, CA, 1985.
[3] A.E. Ruehli and G.S. Ditlow, "Circuit Analysis, Logic Simulation, and Design Verification for VLSI," Proc. of the IEEE, vol. 71, pp. 34-48, January 1983.

[4] J.K. Ousterhout, "A Switch-Level Timing Verifier for Digital MOS VLSI," IEEE Trans. on Computer-Aided Design, vol. CAD-4, pp. 336-349, July 1985.

[5] O. Sorasen, "Design Techniques and Trade Offs for a Full Custom Design of IC's," Proc. of the Custom Integrated Circuit Conference, pp. 117-121, 1980.

[6] A. Vladimirescu, A. R. Newton, and D. O. Pederson, SPICE Version 2G.O User's Guide, University of California, Berkeley, Sept. 1980.

[7] M. A. Samad, "A Toolbox for ASIC Testability Automation," Proc. of the Custom Integrated Circuit Conference, pp. 28.1.1-28.1.4, 1990.

[8] F.F. Tsui, "Problems in Testing" in LSI/VLSI Testability Design, Chapter 3, McGraw-Hill, 1987.

[9] LV500 Operator's Manual, Version 1.60, Tektronix, Inc., 1991.

[10] TekWaves 1.0 User's Guide, Tektronix, Inc., 1991.

[11] F.E. Norrod, "An Automatic Test Generation Algorithm for Hardware Description Language," Proc. of the 26th ACM/IEEE Design Automation Conference, pp. 429-434, 1989.

[12] J.F. Santucci, A.L. Courbis, and N. Giambiasi, "Behavioral Testing of Digital Circuits," J. of Microelectronic System Integration, vol. 1, pp. 55-77, March 1993.

\section{APPENDIX A}

\section{SPICE 2G.6 OUTPUT FILE}

\begin{tabular}{|c|c|c|c|c|c|c|c|}
\hline \multicolumn{8}{|c|}{ OCLOCK GENERATOR } \\
\hline \multicolumn{3}{|c|}{$0 * * * *$} & \multicolumn{5}{|c|}{ INPUT LISTING } \\
\hline \multicolumn{8}{|c|}{$0 * * * * * * * * * * * * * * * * * * * * * * * * * * * * * * * * * * * * * * * * * * * * *$} \\
\hline M1 & 3 & 2 & 1 & 1 & MOD1 & $\mathrm{W}=3 \mathrm{U}$ & $\mathrm{L}=3 \mathrm{U}$ \\
\hline M2 & 6 & 3 & 1 & 1 & MOD1 & $\mathrm{W}=3 \mathrm{U}$ & $\mathrm{L}=3 \mathrm{U}$ \\
\hline M3 & 5 & 4 & 6 & 1 & MOD1 & $\mathrm{W}=3 \mathrm{U}$ & $\mathrm{L}=3 \mathrm{U}$ \\
\hline M4 & 7 & 5 & 1 & 1 & MOD1 & $\mathrm{W}=3 \mathrm{U}$ & $\mathrm{L}=3 \mathrm{U}$ \\
\hline M5 & 4 & 2 & 7 & 1 & MOD1 & $\mathrm{W}=3 \mathrm{U}$ & $\mathrm{L}=3 \mathrm{U}$ \\
\hline M6 & 3 & 2 & 0 & 0 & MOD2 & $\mathrm{W}=3 \mathrm{U}$ & $\mathrm{L}=3 \mathrm{U}$ \\
\hline M7 & 5 & 3 & 0 & 0 & MOD2 & $\mathrm{W}=3 \mathrm{U}$ & $\mathrm{L}=3 \mathrm{U}$ \\
\hline M8 & 5 & 4 & 0 & 0 & MOD2 & $\mathrm{W}=3 \mathrm{U}$ & $\mathrm{L}=3 \mathrm{U}$ \\
\hline M9 & 4 & 5 & 0 & 0 & MOD2 & $\mathrm{W}=3 \mathrm{U}$ & $\mathrm{L}=3 \mathrm{U}$ \\
\hline M10 & 4 & 2 & 0 & 0 & MOD2 & $\mathrm{W}=3 \mathrm{U}$ & $\mathrm{L}=3 \mathrm{U}$ \\
\hline
\end{tabular}

TEMPERA-

TURE =

27.000 DEG C

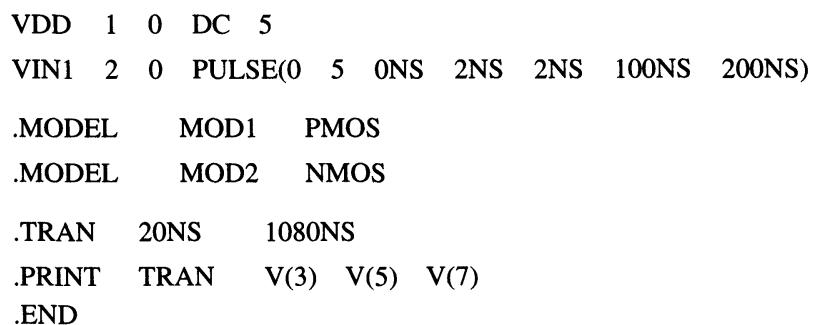




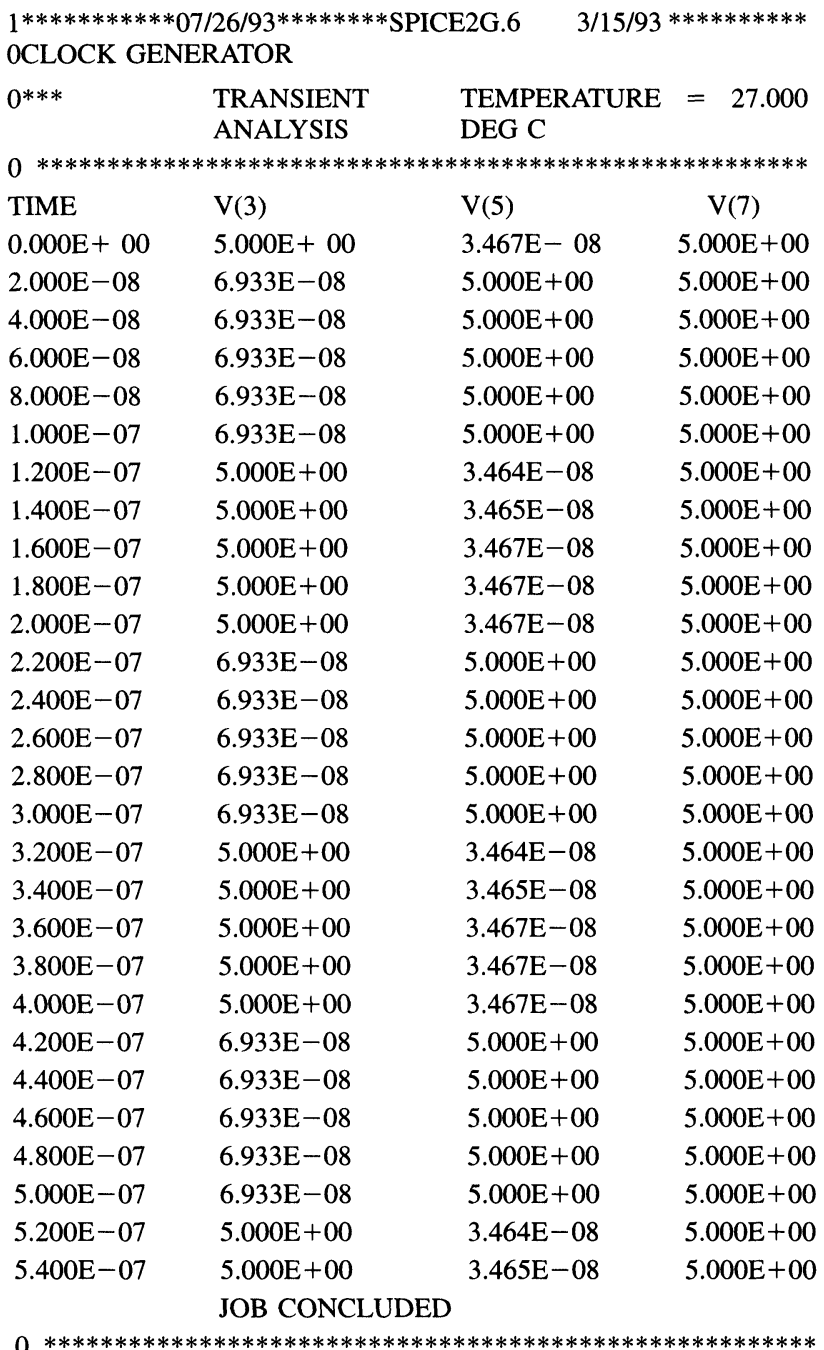

\section{Biographies}

A. SRIVASTAVA has served as a scientist at the Central Electronics Engineering Research Insitutue, Pilani; and on the faculty of Birla Institute of Technology and Science, Pilani, India; North Carolina State University; State University of New York; University of Cincinnati and as a UNESCO Fellow; as a visiting scientist and UNESCO Fellow at the University of Arizona. Currently he is an Associate Professor of Electrical and Computer Engineering at the Louisiana State University in Baton Rouge. His research interests include CMOS/BiCMOS VLSI Design and device modeling, cryogenic CMOS electronics, and smart gas sensors. He is senior member of the IEEE including the IEEE

\section{EWAV FILE}

version event 010 ; date 1119 1992;

time 9406 ;

timescale $=1 \mathrm{~ns}$

signal VIN1 input; signal VIN2 input; signal VIN4 input; signal VIN3 input; signal VIN5 input; signal V3 output; signal V5 output; signal V7 output;

/*RESET Instruction*/ 0: 01010HLH; 20: 00010LHH; 40: $00011 \mathrm{LHH}$ 60: 10111LHH; 80: 10101LHH; $1 *$ Count to $1 * /$ 100: 01011LHH; 120: 00011HLH; 140: 00011HLH; 160: 10110HLH; 180: 10110HLH; $1 *$ Count to $2 * 1$ 200: 01000HLH; 220: 00000LHH; 240: 00001LHH; 260: 10101LHH; 280: 10111LHH; /*Count to $3 * 1$ 300: 01011LHH; 320: 00011HLH; 340: 00011HLH; 360: 10111HLH; 380: 10111HLH; /*Count to $4 * /$ 400: 01011HLH; 420: 00011LHH; 440: 00011LHH; 460: 10111LHH; 480: 10111LHH;

Computer Society and the member of the Cryogenic Society of America. His e-mail address is ashok@gate.ee.lsu.edu.

S.R. PALAVALI has graduated with a M.S. degree in Electrical Engineering from the Louisiana State University, Baton Rouge in 1993. $\mathrm{He}$ has received his B.Tech. degree in Electronics and Communications Engineering from Sri Venkateswara University, India in 1991. Currently he is a software consultant to Electronic Data Systems in Plano, Texas. His research interests include programming in $\mathrm{C}^{++}$in UNIX environment. 

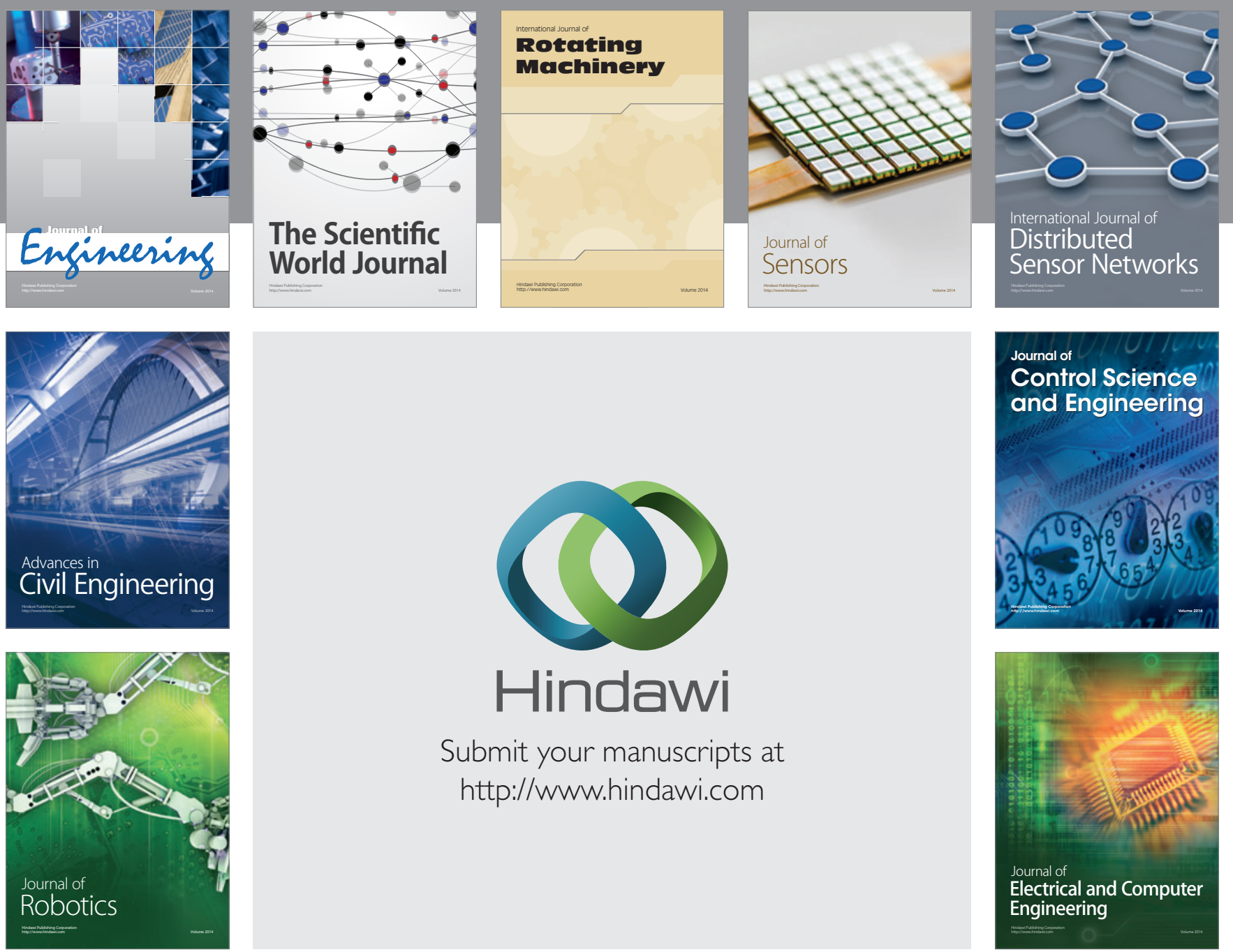

Submit your manuscripts at

http://www.hindawi.com
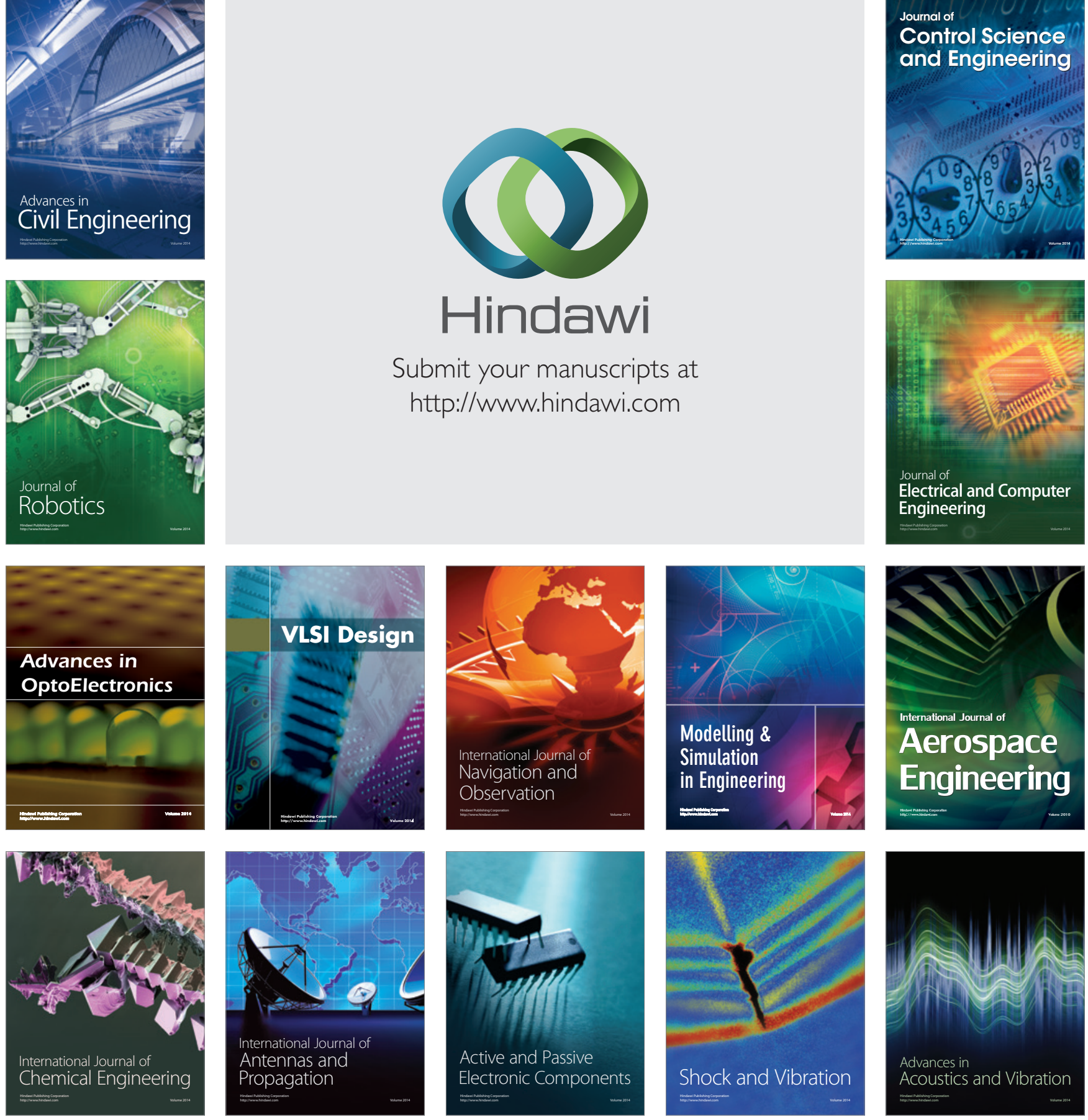